\title{
COMMENT
}

\section{Improving diversity in medical research}

Ashwarya Sharma (i) and Latha Palaniappan ${ }^{\bowtie}$

Clinical research is essential for the advancement of medicine; however, trials often enrol

homogeneous populations that do not accurately represent the patient populations served.

Representative and diverse research participation is necessary to establish fair standards of

care, minimize outcome disparities between populations, and achieve and uphold social equity.
In the future, we must strive to represent all populations that will eventually use the tested drugs and devices 凶e-mail: lathap@stanford.edu https://doi.org/10.1038 s41572-021-00316-8
Since the early 2000s, clinical research has become more global and complex, as the number of clinical trials conducted to create interventions that improve patients' health is increasing worldwide. Interest in the community is growing to recognize unique characteristics of clinical trial participants, in an attempt to better understand variability in drug responses. However, the diversity of patient populations remains under-represented in clinical trials, despite being a vital component in enabling medical research to move towards precision medicine approaches.

Severe imbalance in the representation of minorities is not new; clinical research has long been criticized for enrolling homogeneous populations that do not accurately represent the communities served.

In a 2020 analysis of the global participation in clinical trials, the FDA highlighted the vast difference between the enrolled participants and the global population. Of 292,537 participants in clinical trials globally, $76 \%$ were white, $11 \%$ were Asian and only $7 \%$ were Black ${ }^{1}$. In comparison, the global population ( $\sim .8$ billion) is distributed with $\sim 60 \%$ of the population in Asia, $~ 16 \%$ in Africa, $\sim 10 \%$ in Europe and $\sim 8 \%$ in Latin America (World Population Review). Similarly, a review of 379 clinical trials funded by the US National Institute of Mental Health published in 1995-2004 found that all racial or ethnic groups except white individuals and African Americans were under-represented, and only $\sim 48 \%$ of the studies provided complete racial or ethnic information ${ }^{2}$. Thus, data for global populations are lacking, and current guidelines and clinical decisions are based on insufficiently diverse trials and studies.

Past thinking has favoured the enrolment of individuals with similar characteristics to limit heterogeneity and to decrease the effects of interindividual variability and achieve consistent short-term results. In the future, we must strive to represent all populations that will eventually use the tested drugs and devices. Although enrolling diverse populations may initially bring higher variability in results than enrolling homogeneous populations, the outcome data can be leveraged through statistical analysis and novel study designs to tailor and individualize therapies, which can ultimately result in improved generalizability for the populations we serve.

Development of interventions that are not tested in diverse populations can lead to treatments that are less effective and less trusted in some populations, despite their need for the intervention. For instance, 5-fluorouracil, a well-studied, commonly used chemotherapeutic drug, was found to lead to adverse effects, including haematological toxicities, in certain individuals. These toxic effects occurred at higher rates in African American individuals than in white individuals ${ }^{3}$. However, this observation was not revealed in preceding clinical trials, as these had limited patient diversity, which ultimately negatively affected African American individuals' health care ${ }^{4}$. Similarly, Ninlaro (ixazomib), approved by the FDA in 2015 for the treatment of multiple myeloma, had only $1.8 \%$ Black participants in the phase III clinical trial despite African Americans having higher incidence and prevalence of this disease than European Americans 5,6 . Under-represented populations are deeply affected by these inequities, as they can lead to distrust and worse health outcomes for certain populations compared with others.

This failure for meaningful diversity in health research also has considerable social and ethical implications, as individuals and entire subgroups that are traditionally under-studied may be unable to access potentially beneficial research. Overall, the imbalance leads to substantial differences in their lifelong care, leading to additional health inequities. Notably, the COVID-19 pandemic has further exposed these great inequalities in health, as Black, Latinx, Pacific Islander and other vulnerable populations have been disproportionately affected by SARS-CoV-2. A 2020 study showed that 34\% of overall deaths were among non-Latinx Black people, although this group only accounts for $13 \%$ of the overall US population? This increased disease mortality in these populations is thought to be due to pre-existing comorbidities, such as hypertension or diabetes, decreased access to testing, inequities in health-care delivery, exposure risks and, potentially, genetic differences. However, the relative risk of any of these underlying factors is unknown, as data addressing these issues are lacking. 


\begin{tabular}{|c|c|c|c|c|}
\hline $\begin{array}{l}\text { Public policy } \\
\text { - Set global standards for } \\
\text { diversity in clinical trials } \\
\text { - Adopt international guidelines } \\
\text { requiring representation of } \\
\text { diverse populations for } \\
\text { regulatory approvals } \\
\text { - Enact continuous post- } \\
\text { marketing surveillance to } \\
\text { monitor effectiveness in } \\
\text { diverse populations }\end{array}$ & $\begin{array}{l}\text { Community } \\
\text { - Involve patients and } \\
\text { communities in the } \\
\text { development of } \\
\text { study questions } \\
\text { - Ensure the intended } \\
\text { population can be } \\
\text { reached with the } \\
\text { planned study } \\
\text { recruitment methods }\end{array}$ & $\begin{array}{l}\quad \text { Institutional } \\
\text { - Develop knowledge } \\
\text { resources specific } \\
\text { to communities } \\
\text { with historical } \\
\text { medical mistrust } \\
\text { - Provide data for } \\
\text { drug efficacy } \\
\text { across different } \\
\text { populations }\end{array}$ & $\begin{array}{l}\text { Interpersonal } \\
\text { - Ensure diversity in research } \\
\text { and development teams } \\
\text { - Plan and track inclusion of } \\
\text { diverse populations through- } \\
\text { out the discovery cycle } \\
\text { - Collect sociodemographic data } \\
\text { of study populations in trials } \\
\text { using uniform data standards }\end{array}$ & $\begin{array}{l}\text { Intrapersonal } \\
\text { - Understand individual } \\
\text { knowledge, beliefs and } \\
\text { attitudes towards clinical } \\
\text { research } \\
\text { - Provide support to encourage } \\
\text { research participation, such } \\
\text { as transportation and } \\
\text { financial assistance }\end{array}$ \\
\hline
\end{tabular}

Fig. 1 Socioecological framework to increase diversity in clinical research. Changes to public policy, community, institutional, interpersonal and intrapersonal domains can result in increased diversity in research and help overcome inequalities in health care and patient outcomes.

Historically, five major challenges have been highlighted for reduced participation in clinical trials - low income; investigator bias; mistrust in medical research and professionals; limited health and research literacy; and lack of access to transportation ${ }^{8}$. In particular, investigator bias and medical mistrust are uniquely present in medical practice. Investigator bias or the implicit biases that health-care providers may have can interfere with enrolment in clinical studies and are associated with poor quality of care. Taken together, patients of racial or ethnic minority have been found to receive poorer care than white patients across numerous illnesses, in part owing to biases and a lack of research on how specific diseases may uniquely affect various populations ${ }^{8}$. For instance, studies have shown that Asian American individuals are more likely to develop diabetes mellitus at lower body weight than white Americans ${ }^{9}$. However, limited resources of health information and research dedicated specifically to Asian American audiences are available. Mistrust and skepticism of medical professionals and the health-care system by minority and other under-represented groups exist owing to historical abuses, such as the US Public Health Service (USPHS) Syphilis Study at Tuskegee and forced sterilization of American Indians. As a consequence, the affected communities have less participation in trials and, in some cases, poorer health outcomes ${ }^{10}$.

To overcome the long-standing inequalities in health care and patient outcomes, the research community must commit to diversity and inclusion in clinical research. Here, we provide a framework for increasing diversity in clinical trials using the socioecological model (FIG. 1). Changes to public policy, community, institutional, interpersonal and intrapersonal domains can be used to increase diversity in research. At the public policy level, we can set strict requirements for representation of diverse populations as a necessity for approval of new drugs and devices. Uniform standards across research are needed to collect and record variables that capture various aspects of diversity, such as race or ethnicity, ancestry, language, religious practices and sexual orientation. At the community level, researchers must consider the specific priorities of patients and communities affected by the condition to ensure that the intended populations can be effectively recruited.
At the institutional level, we must develop knowledge resources specifically for communities with historical medical mistrust. Institutions can transparently provide data for drug efficacy across different populations and acknowledge and address areas in which data do not currently exist. At the interpersonal level, we need to increase representation across training pathways to ensure diversity in all research and development teams. Further work is needed to understand knowledge, beliefs and attitudes towards clinical research at the intrapersonal level and known barriers to involvement in research should be addressed with the required support measures.

The imbalance of representation of diverse groups in clinical research is a problem that continues to adversely affect health care for all and is one that medical professionals must be prepared to address.

1. U.S. Food \& Drug Administration. 2015-2019 drug trials snapshots summary report. FDA https://www.fda.gov/media/143592/download (2020).

2. Mak, W. W. S. et al. Gender and ethnic diversity in NIMH-funded clinical trials: review of a decade of published research. Adm. Policy Ment. Health 34, 497-503 (2007)

3. McCollum, A. D. et al. Outcomes and toxicity in African-American and caucasian patients in a randomized adjuvant chemotherapy trial for colon cancer. J. Natl Cancer Inst. 94, 1160-1167 (2002).

4. Meta-Analysis Group In Cancer et al. Toxicity of fluorouracil in patients with advanced colorectal cancer: effect of administration schedule and prognostic factors. J. Clin. Oncol. 16, 3537-3541 (1998).

5. U.S. Food \& Drug Administration. Drug trials snapshots: ninlaro. FDA https://www.fda.gov/drugs/drug-approvals-and-databases/ drug-trials-snapshots-ninlaro (2016).

6. Landgren, O. et al. Risk of monoclonal gammopathy of undetermined significance (MGUS) and subsequent multiple myeloma among African American and white veterans in the United States. Blood 107, 904-906 (2006).

7. Holmes, L. Jr. et al. Black-white risk differentials in COVID-19 (SARS-COV2) transmission, mortality and case fatality in the United States: translational epidemiologic perspective and challenges. Int. J. Environ. Res. Public Health 17, 4322 (2020).

8. Bierer, B. E. et al. Achieving diversity, inclusion, and equity in clinical research guidance document version 1.2. The MRCT Center of Brigham and Women's Hospital and Harvard https://mrctcenter.org/ diversity-in-clinical-research/download/2326/ (2021).

9. Hsu, W. C. et al. BMI cut points to identify at-risk Asian Americans for type 2 diabetes screening. Diabetes Care 38 150-158 (2015)

10. George, S., Duran, N. \& Norris, K. A systematic review of barriers and facilitators to minority research participation among African Americans, Latinos, Asian Americans, and Pacific Islanders. Am. J. Public Health 104, e16-e31 (2014).

\section{Competing interests}

The authors declare no competing interests.

RELATED LINKS

World Population Review: https://worldpopulationreview.com/continents 